\section{Severe orthostatic hypotension due to unilateral carotid artery dissection}

\author{
Nobuyuki Ishii, Hitoshi Mochizuki, \\ Akitoshi Taniguchi, Kazutaka Shiomi, \\ Masamitsu Nakazato \\ Division of Neurology, Respirology, \\ Endocrinology and Metabolism, \\ Department of Internal Medicine, \\ University of Miyazaki, Japan
}

\section{Abstract}

A 46-year-old woman was admitted to our hospital with mild right-sided hemiparesis and orthostatic hypotension. Magnetic resonance angiography of the neck showed stenosis of the left distal carotid sinus surrounded by intraluminal hyperintensities on both T1weighted and T2-weighted images, representing a periluminal hematoma secondary to carotid artery dissection. The dissection hyperextended the carotid artery wall and stimulated baroreceptors in the carotid sinus. The stimulated baroreceptors induced carotid sinus hypersensitivity, which may have been related to her orthostatic hypotension. Poststroke orthostatic hypotension should prompt consideration of carotid artery dissection.

\section{Introduction}

Orthostatic hypotension, syncope, or both, sometimes occur in patients with cerebrovascular stroke.1 Carotid artery dissection is one possible cause of these symptoms, but the underlying mechanism remains debated. ${ }^{2-4} \mathrm{We}$ report a case of severe orthostatic hypotension after unilateral internal carotid artery dissection.

\section{Case Report}

A 46-year-old woman was admitted to our hospital with mild right-sided hemiparesis and orthostatic hypotension. One day before admission, she experienced an abrupt blurred vision in the left eye, lightheadedness and mild headache while driving. This blurred vision continued for 5 to $10 \mathrm{~min}$. 0 n the next day, right-sided weakness and orthostatic hypotension developed, and she was admitted to our hospital.

On admission, her general medical physical examination findings were normal. Her height was $147 \mathrm{~cm}$ and she weighted $46 \mathrm{~kg}$. Neurological examination showed mild rightsided hemiparesis and orthostatic hypotension (positive tilt test; Table 1). She did not have findings associated with Horner's syndrome or any cervical bruits. She had no past medical histories and no previous episodes of orthostatic hypotension before this admission. Initial routine laboratory examinations were unremarkable. Diffusion-weighted and T2-weighted magnetic resonance imaging (MRI) of the brain showed a hyperintense lesion in the genu of the left internal capsule and globus pallidus (Figure 1A,B). MR angiography (MRA) of the head was normal; MRA of the neck showed stenosis of the left distal carotid sinus surrounded by intraluminal hyperintensities on both T1-weighted and T2-weighted images, representing a periluminal hematoma secondary to carotid artery dissection (Figure 1C-G).

The patient's blood pressure and heart rate over her clinical course are shown in Table 1. She was lying back during the first day of admission. A tilt test revealed severe orthostatic hypotension on the second day of admission. The coefficient of variation of the RR intervals (CVRR) was decreased. The patient was diagnosed with severe orthostatic hypotension and cerebral infarction secondary to internal carotid artery dissection, and medicated with aspirin $100 \mathrm{mg} /$ day. After two weeks, her right hemiparesis was resolved with rehabilitation therapy, but orthostatic hypotension persisted despite treatment with amezinium $20 \mathrm{mg}$ daily. On 110th day after admission, MRI of the neck showed decreased stenosis of the left carotid artery (Figure 1H,I). On 152nd day after admission, her orthostatic hypotension improved, and the tilt test became negative.

\section{Discussion}

One day before admission, she experienced a temporal visual disturbance during driving. Since this symptom and headache occurred at the similar time, this symptom would be a temporal retinal ischemia due to a thrombosis from carotid artery dissection.

Post-stroke patients sometimes present with orthostatic hypotension, syncope, or both. The right insula controls sympathetic cardiovascular function, so an impairment of this area may cause bradyarrhythmias. ${ }^{5}$ Since our patient's lesion did not involve the insular cortex, her severe orthostatic hypotension could be related to carotid artery dissection. Carotid sinus hypersensitivity (CSH), which involves hypotension and bradycardia after carotid stenting, ${ }^{6}$ is a well-recognized entity that is sometimes accompanied by orthostatic
Correspondence: Hitoshi Mochizuki, Division of Neurology, Respirology, Endocrinology and Metabolism, Department of Internal Medicine, University of Miyazaki, 5200 Kihara, Kiyotake, Miyazaki, 889-1692, Japan.

Tel. +81.985.85.2965 - Fax: +81.985 .85 .1869 .

E-mail: mochizuki-h@umin.ac.jp

Key words: orthostatic hypotension, carotid artery dissection, infarction, carotid sinus hypersensitivity.

Contributions: the authors contributed equally.

Conflict of interests: the authors declare no potential conflict of interests.

Received for publication: 13 February 2014.

Revision received: 27 March 2014.

Accepted for publication: 28 March 2014.

This work is licensed under a Creative Commons Attribution NonCommercial 3.0 License (CC BYNC 3.0).

(C) Copyright N. Ishii et al., 2014

Licensee PAGEPress, Italy

Neurology International 2014; 6:5352

doi:10.4081/ni.2014.5352

hypotension. ${ }^{7}$ In our patient, heart rate and blood pressure decreased as compared with this admission before (Table 1). Her symptoms, including orthostatic hypotension, could have been caused by $\mathrm{CSH}$.

The carotid sinus reflex arc is composed of an afferent limb that arises from mechanoreceptors in the internal carotid artery and terminates in the vagal nucleus and the vasomotor center in the midbrain. ${ }^{8 \mathrm{CSH}}$ is an exaggerated response of the carotid sinus baroreceptors to local stimulation, which can be due to changes in either parts of the reflex arc or the target organs. CSH resulting from local factors that exert pressure on the carotid sinus such as tumors, lymph nodes, radiotherapy, and scars has been described.9,10 CSH induced by carotid artery dissection have been reported only in two cases. ${ }^{2,4}$ In one case, CSH was associated with bilateral carotid artery dissection. ${ }^{4}$ In the other case, there was a large cerebral infarct involving the insula. ${ }^{2}$ To our knowledge, our case is the first one of $\mathrm{CSH}$ due to unilateral carotid artery dissection. Stenosis in the distal part of the carotid sinus has been reported as a cause of $\mathrm{CSH}$ and orthostatic hypotension. ${ }^{7}$ In our patient, continuous stimulation of baroreceptors due to the stenosis caused by the carotid arterial dissection could have induced CSH, orthostatic hypotension, and hyperactivity of the parasympathetic nervous system as indicated by low CVRR values. 
Table 1. Blood pressure, heart rate, tilt test and coefficient of variation of the R-to- $R$ intervals values.

\begin{tabular}{lccc} 
Values & Before admission & 2nd day after admission & 152nd day after admission** \\
Blood pressure (mmHg) & $100-120 / 60-80$ & $90-110 / 60-80$ & $90-110 / 60-80$ \\
Heart rate (bpm) & around 80 & $60-70$ & $80-90$ \\
\hline Tilt test & NPE & $110 / 74 \mathrm{mmHg}, 68 / \mathrm{min}$ & $109 / 64 \mathrm{mmHg}, 89 / \mathrm{min}$ \\
Supine & & $55 / 33 \mathrm{mmHg}, 68 / \mathrm{min}$ & $83 / 51 \mathrm{mmHg}, 95 / \mathrm{min}$ \\
Upright & - & 1.39 & 2.44 \\
CVRR (\%) & & & \\
\hline
\end{tabular}

*Amezinium $10 \mathrm{mg} /$ day; NPE, no previous episodes of orthostatic hypotension; CVRR, coefficient of variation of the R-to-R intervals.
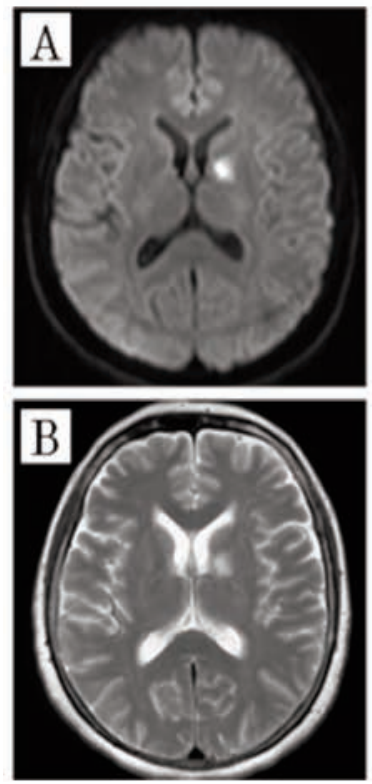
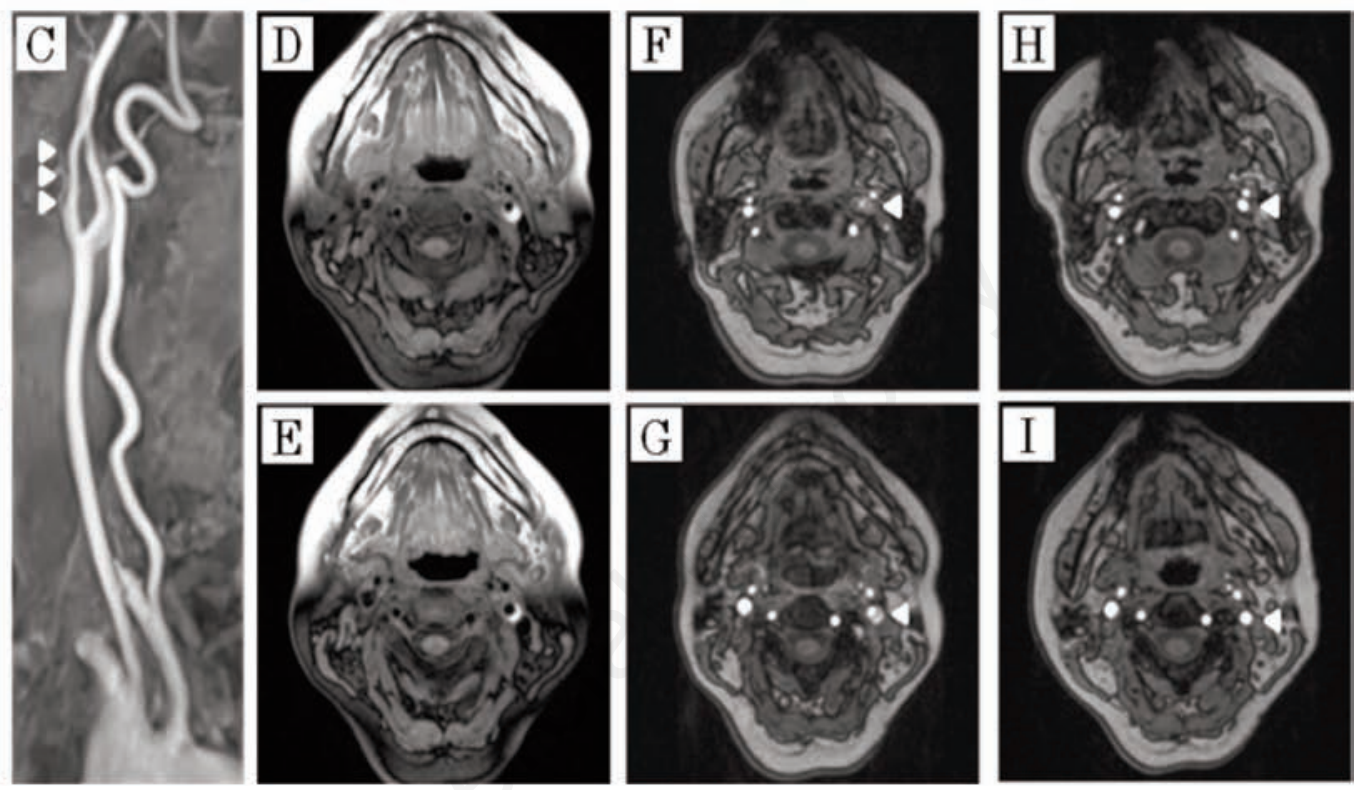

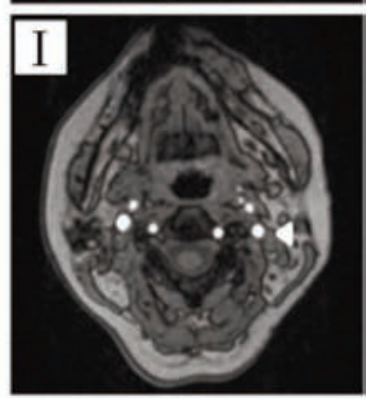

Figure 1. Diffusion-weighted (A) and T2-weighted (B) magnetic resonance imaging (MRI) of the brain shows a hyperintense lesion in the genu of the left internal capsule and globus pallidus. Magnetic resonance angiography (MRA) of the neck reveals narrowing of the left distal carotid sinus (C, arrowheads) surrounded by hyperintensities seen on both T2-weighted (D,E) and T1-weighted (F,G, arrowhead) sequences, which represent a periluminal hematoma secondary to carotid artery dissection. On 110th day after admission, MRI of the neck showed less severe stenosis of her left carotid artery (H,I, arrowhead).

\section{Conclusions}

In this communication, we present the first case of severe orthostatic hypotension due to unilateral internal carotid artery dissection without insular injury. Post-stroke orthostatic hypotension should prompt consideration of carotid artery dissection. Even if clinically overt signs of stroke did not exist, the presence of an orthostatic hypotension might be a sign of a carotid artery dissection.

\section{References}

1. Kelley RE, Minagar A. Cardioembolic stroke: an update. South Med J
2003;96:343-9.

2. Dulay D, Gould PA, Leung A, Krahn AD. Images in cardiovascular medicine. A sensitive dissection: profound bradycardia complicating carotid dissection. Circulation 2008;118:e152-3.

3. Sarikaya H, Baumgartner RW, Arnold M. Letter by Sarikaya et al. regarding the article "A sensitive dissection: profound bradycardia complicating carotid dissection". Circulation 2009;119:e545-6.

4. Carruthers R, Ropper A, Cohen AB. Dysautonomia from bilateral carotid artery dissection. Circulation 2011;123:3009-11.

5. Oppenheimer S. Cerebrogenic cardiac arrhythmias: cortical lateralization and clinical significance. Clin Auton Res 2006;16:6-11.

6. Mlekusch W, Schillinger M, Sabeti S, et al.
Hypotension and bradycardia after elective carotid stenting: frequency and risk factors. J Endovasc Ther 2003;10:851-61.

7. Akiyama Y, Hashimoto N, Morimoto M. Orthostatic hypotension improved after bilateral carotid endarterectomy - case report. Neurol Med Clin (Tokyo) 1999; 39:153-6.

8. Thomas JE. Hyperactive carotid sinus reflex and carotid sinus syncope. Mayo Clin Proc 1969;44:127-39.

9. Badin RW, Panje WR. The incidence of vasovagal reflex activity during radical neck dissection. Laryngoscope 1980;90: 1321-3.

10. Strasberg B, Sagie A, Erdman S, et al. Carotid sinus hypersensitivity and the carotid sinus syndrome. Prog Cardiovas Dis 1989;31:379-91. 